late 30 s would have traversed almost none of the risk period. Although there are a number of deficiencies outlined by the authors with their high-risk strategy, there are some limitations which they do not outline. The actual risk to illness differs substantially across individual subjects.

To my surprise, the authors never precisely define what they mean by 'well'. In the middle of the 'Results' section, they do make the statement that "no one in either group reached the criteria of a current psychiatric disorder in the PSE or SADS-L". Is that their definition? If someone had a prior episode of psychosis or depression or alcohol dependency from which they recovered and are no longer symptomatic, does that render them well? What about an individual who has four of the nine criteria for schizotypal personality disorder. Would this person be considered 'well'?

The statement on page 547 of the risk for schizophrenia in children of one parent with schizophrenia could hardly be cited in such a definitive way. There is a range of risks and the most recent empirical risk figures from the New York and Copenhagen high-risk studies are different from the summary results presented here. Curiously, they discuss preliminary data supported by Kendler et al on the Structured Interview for Schizotypy, but do not cite or comment on complete analysis of the schizotypal symptoms and signs in the Rosscommon Family Study (Kendler et al, 1995).

Kendilor, K. S., McGulre, M., Gruenbers, A. M., it of (1995) Schizotypal symptoms and signs on the Roscommon Family Study: their factor structure and familial relationship with psychotic and affective disorders. Archives of Generol Psychiotry, 52, 296-303.

Stromgren, E. (1935) Zum Ersatz des Weinbergschen "Abgekurzten Verfahrens". Zugleich ein Beitrag zur Frage von des Erblichkeit des Erkrankungsalters bei der Schizophrenie. Zeitschrift Gesomte für Neurologie und Psychiotrie, 153, 784-797.

Professor Kenneth S. Kendler Department of Psychiatry, Virginia Institute for Psychiatric and Behavioral Genetics, Medical College of Virginia Commonwealth University, 800 East Leigh Street, PO Box 980126 Richmond, Virginia 23298-0126,USA

\section{NEED FOR MORE RIGOROUS PRELIMINARY REPORTING}

The study describes the design, methods and selected 'baseline' characteristics in a set of 'high-risk' subjects representing
$50 \%$ of the projected sample size of 100 , and in 30 control individuals. The study is prospective by design. The authors also intend to include 30 patients with 'sporadic' schizophrenia. Apart from demographic data, the manuscript includes items from previous history (psychiatric and forensic) and a selection of scores from the Structured Interview for Schizotypy (SIS).

This is a potentially important study which takes a novel approach to the study of risk factors for schizophrenia. However, there is little in this manuscript to justify its publication, since none of the preliminary findings about this half of the sample contributes any new substantive knowledge about the precursors or risk factors in schizophrenia. Hopefully, such knowledge will be forthcoming. The authors should have considered a shorter, tightly written preliminary report outlining more clearly the design of the study and the main background variables describing the study population. Some specific questions that should have been addressed are: (a) Were the SIS interviews conducted blind to the high-risk/control status? (b) How many individuals met the DSM-IV or ICD-10 criteria for schizotypal disorder? A table giving a breakdown of the sample by number of affected family members and degree of relatedness (i.e. affected sibling pairs, parent-sibling, etc.) could have been included as well as a table listing the neuropsychological assessments and the magnetic resonance imaging measures being collected.

Professor Assen Jablensky Department of Psychiatry and Behavioural Science. University of Western Australia, Medical Research Foundation Building, Level 3, Rear 50 Murray Street, Box $\times 2213$ GPO, Perth, WA 600I, Australia

\section{FAMILIES WITH A GENETIC 'TAINT' OR THE TIP OF UBIQUITOUS VARIATION FOR THE HUMAN CAPACITY FOR LANGUAGE?}

At first evaluation it seems as though the findings of this study are predictable. Psychiatric illness, forensic contacts and delinquency are higher in the at-risk group than the control group. This could be genetic predisposition or it could presurnably be reactive to illness in the family. The increase in premorbid personality anomalies (social isolation, restricted affect etc.) is perhaps more likely to reflect the genetic predisposition. It is consistent with the findings from cohort studies.

The conclusions the authors draw are that the differences may represent increased risk but "their true significance will not be revealed until the cohort has been followed through the at-risk years". An unsympathetic reader might conclude 'let's wait for the full analysis and see - there's no justification for publication at this stage'. Even so, one can ask whether, if the conclusions are relatively predictable at this stage, much more will be achieved with a larger sample and a longer follow-up. Is it not likely that the group who develop a psychotic illness will be more abnormal on these same indices than those who do not? That is, there will be quantitative deviations along the axes of abnormal behaviour and 'schizotypy'.

This question is worth asking because a salient feature of the paper (and maybe the study) is the absence of hypotheses about the nature of the genetic predisposition and the nature of the illness. That there is a category of illness that can be readily isolated and labelled schizophrenia is taken as read (the criteria adopted are not mentioned in the summary), but this is doubtful (see Endicott et al, 1982). There are different criteria and there is almost certainly a spectrum or continuum of illness (see Crow, 1994, 1995).

These considerations are no doubt well known to the investigators, but they may be relevant to the way the analysis of the study proceeds in the future. Thus, this background has had no impact on the rather naive genetic models presented. A further important point (relevant to the issue of the survival of genetic predisposition, considered in the 'Discussion') is uniformity of incidence across populations. As has been argued in the papers cited above, this finding, which has now to be considered relatively securely established, has had no impact on the psychiatric genetic literature or on genetic models. It must mean that predisposition to schizophrenia is a part of variation that crosses the population as a whole. It is the nature of this variation - what are the critical dimensions of variation of which psychosis is the extreme-that is the key question. It seems as though the data in this study, particularly when considered in conjunction with the structural and psychometric studies which, although not mentioned here, are presumably a major part of the 